\title{
Haemostatic Abnormalities in Patients with Cirrhosis and their Relation with Severity of Liver Dysfunction as Assessed by Child Pugh Score
}

\author{
Hanozia Hassan, Asmat Kamal Ansari, Salma Farrukh Memon
}

\section{ABSTRACT}

OBJECTIVE: To evaluate the hemostatic abnormalities in patients with cirrhosis and their relation with severity of liver dysfunction as assessed by Child Pugh Score.

BACKGROUND: Important role is played by liver in hematopoeisis as well as hemostasis. Therefore liver dysfunction due to any reason is associated with hematological and complex hemostatic abnormalities. Important abnormalities include decreased production of coagulation and anticoagulation factors, excessive fibrinolysis, low platelet count etc. These are manifested by abnormal laboratory tests for these variables. For descriptive or prognostic indicator of these abnormalities Child- Pugh score is generally used.

MATERIAL AND METHODS: Present study was done at the Postgraduate laboratory and hospital of ISRA University Hyderabad. The study was approved by ethical committee of ISRA University. Sixty four patients with liver cirrhosis were taken for study and divided according to Child Pugh Score as Class A, $B$ and C; 47 were males and 17 were females. All age groups were included and mean age of group A, B and $C$ was determined.

The patients fulfilled inclusion and exclusion criteria. Data was collected after taking consent and blood was drawn to perform Coagulation parameters, platelets and fibrinolytic parameters in their respective analyzer machines.

RESULTS: In Child Pugh Score A, B and C patients, Mean Platelet count was $375.36 \pm 39.479,343.30 \pm$ 58.793, $277.03 \pm 55.061$ per $u L$ and Mean Fibrinogen level was $333.90 \pm 45.007,261.07 \pm 70.622,207.33 \pm$ 38.484 per $\mathrm{mg} / \mathrm{dl}$ respectively which showed a significant decrease in patients from Child Pugh Score $A$ through $C$ and Mean Platelet count and Mean Fibrinogen level Showed a negative Correlation with Child Pugh Score having $r-=-0.640, p=0.0001$ and $r-=-0.579, p 0.0001$ respectively. In Child Pugh Score A, B and C patients, Mean Prothrombin time was 11.09 $\pm 0.831,12.15 \pm 2.828,16.66 \pm 2.541$ per second, Mean Activated partial thromboplastin time was 27.09 \pm 4.657, $33.50 \pm 2.284,35.62 \pm 1.779$ per second, Mean D-dimer level was $195.45 \pm 13.633,212.96 \pm 37.845,234.14 \pm 47.287$ per ug/L and Mean bleeding time was $4.63 \pm 1804,7.88 \pm 2.833,9.33 \pm 2.496$ per minute respectively which showed a significant increase in patients from class $A$ through $C$ and showed a positive correlation with Child Pugh score.

CONCLUSION: It was concluded that PT, APTT, BT and D-dimer correlated positively while Fibrinogen level and Platelet count correlated negatively with Child Pugh score.

KEY WORDS: Child Pugh Score, Fibrinogen, PT, APTT, D-dimer.

This article may be cited as: Hassan H, Ansari AK, Memon SF. Haemostatic Abnormalities in Patients with Cirrhosis and their Relation with Severity of Liver Dysfunction as Assessed by Child Pugh Score. J Liaquat Uni Med Health Sci. 2017;16(02): 108-13.

doi: $10.22442 /$ jumhs. 171620516

\section{INTRODUCTION}

Chronic hepatocellular injury leads to liver cirrhosis that is characterized by both fibrosis and nodular regeneration. Hepatocyte dysfunction, portal hypertension and porto-systemic shunting accounts for most of its clinical features ${ }^{1}$. Cirrhosis can occur at any age, has sufficient morbidity and is an important cause of premature death ${ }^{2}$. Pathological features of cirrhosis are regenerating nodules segregated by fibrous septa and absence of the normal lobular architecture inside nodules $^{3}$. Liver has important role in all steps of hemostasis. It is the place of production of almost all coagulation factors and inhibitors as well as removal of activated coagulation factors and enzyme inhibitor complexes ${ }^{4}$.

In cirrhosis there is imbalance between clotting and anti-clotting mechanisms leading increased risk of 
thrombosis as well as bleeding ${ }^{5-7}$. Patients are usually hospitalized for several complications related to liver disease and matters of hemostasis or coagulation usually affect their course ${ }^{8}$.

Child-Pugh classification involves total bilirubin, albumin, international normalized ratio (INR) or Prothrombin time, hepatic encephalopathy and Ascites. It is very frequently used scoring system for judging the prognosis of cirrhosis of liver ${ }^{9}$. Every measure is scored $1-3$ and 3 indicates very intense imbalance.

Present study was undertaken to assess haemostatic abnormalities in cirrhotic patients and their relation with severity of liver dysfunctions as assessed by Child Pugh Score.

\section{MATERIAL \& METHODS}

Present study was done at the postgraduate laboratory and hospital of ISRA University Hyderabad after ethical approval. This study included 64 patients of cirrhosis of liver; both genders included, regardless of age. These were selected from various Medicine wards and OPD of ISRA Hospital, Hyderabad. Informed consent was taken from all the patients.

Main criterion of inclusion was the existence of cirrhosis of liver, regardless of etiology. Diagnosis of cirrhosis was based upon clinical features, laboratory tests and ultrasound abdomen.

Exclusion criteria included Known case of Coagulation disorder, persons taking drugs that interfere with coagulation parameters e.g. oral contraceptive, aspirin, heparin, and warfarin, patients with their liver disorders other than the cirrhosis and expected women.

Sampling Technique: Non- Probability sampling. Duration of study: 6 months from $15^{\text {th }}$ May 2015 $15^{\text {th }}$ Nov 2015

\section{Data Collection Procedure}

Consent form was given to patients and patients were informed regarding the procedure and were undersigned by the patient, $5 \mathrm{ml}$ blood was taken from ante cuboidal fossa and placed in respective bottles then the blood samples were analyzed for following lab tests:

- Plasma Prothrombin time (PT) by CA-50 SYSMEX MACHINE.

- Activated partial thromboplastin time (APTT) by CA-50 SYSMEX MACHINE

- Plasma fibrinogen level by Automatic Coagulation analyzer.

- Fibrin degradation products (D-dimer) by COBAS $\mathrm{H} 232$.
- Platelet count by CBC analyzer.

- Bleeding time was done manually.

- Total bilirubin By 902 HITACHI AUTOMATIC ANALYZER

- Serum albumin By 902 HITACHI AUTOMATIC ANALYZER

The selected patients were divided into 3 classes according to Child Pugh Score.

\section{Statistical Analysis}

The data obtained was analyzed through SPSS 21.0, P-value of $<0.05$ was taken as statistically significant. ANOVA and Pearson's Co-relation test were applied.

\section{RESULTS}

The patients were divided into three classes according to Child Pugh score on the basis of Ascites, encephalopathy, serum bilirubin levels and serum albumin level. Class A had 11, class B had 26 and class $C$ had 27 patients.

Results are summarized in tables 1-4.

Table-1 shows Prothrombin time and Activated partial thromboplastin time in three groups. PT showed a significant difference between Child Pugh class $B$ and $C(p=0.0001)$ and Child Pugh class $A$ and $C(p=$ $0.0001)$ respectively. Activated partial thromboplastin time was significantly different in these groups.

Table-2 shows mean fibrinogen value \& D-dimer levels in three groups and there was significant difference in the values in these groups. D-dimer values show a significant difference is observed between Child Pugh Score A and C $(p=0.022)$

Table-3 shows mean platelet count and bleeding time in three groups. Platelet count show a significant difference between Child Pugh class $B$ and $C$ $(p=0.0001)$ and Child Pugh class $A$ and $C(p=0.0001)$ respectively, Bleeding time show a significant difference is observed between Child Pugh class $A$ and $B$ $(p=0.002)$ and Child class $A$ and $C(p=0.0001)$ respectively.

Table-4 Child Pugh score in correlation with various parameters. Prothrombin time $(r-=0.664)$ $(p=0.0001)$, Activated partial thromboplastin time $(r-$ $=0.711)(p=0.0001)$, D-dimer level $(r-0.348)(p$ $0.005)$ and Bleeding time $(r-0.533)(p=0.0001) \quad$ (Fig. IV-10 to IV-13 respectively) were positively correlated while Fibrinogen level $(r-=-0.640)(p=0.0001)$ and Platelet count $(r-=-0.579)(p=0.0001)(F i g$. IV-14 to IV-15 respectively) were negatively correlated. 
TABLE I: PROTHROMBIN TIME (SEC) AND

PARTIAL THROMBOPLASTIN TIME (SEC) IN CHILD CLASS A, B AND C $(n=64)$

\begin{tabular}{|c|c|c|}
\hline Child Pugh Score & MEAN \pm S.D & ${ }^{*} p$ \\
\hline \multicolumn{3}{|c|}{ Prothrombin time } \\
\hline Class A ( $n=11)$ & $11.09 \pm 0.831$ & A vs $B \quad 0.464$ \\
\hline Class B $(n=26)$ & $12.15 \pm 2.838$ & B vs C 0.0001 \\
\hline Class C $(n=27)$ & $16.66 \pm 2.541$ & A vs C 0.0001 \\
\hline \multicolumn{3}{|c|}{ Activated partial thromboplastin time } \\
\hline Class A $(n=11)$ & $27.09 \pm 4.657$ & A vs $B \quad 0.0001$ \\
\hline Class B $(n=26)$ & $33.50 \pm 2.284$ & B vs C 0.013 \\
\hline Class C $(n=27)$ & $35.62 \pm 1.779$ & A vs C 0.0001 \\
\hline
\end{tabular}

TABLE II: FIBRINOGEN LEVEL (MG/DL), AND D-DIMER LEVEL (UG/LIT), PLATELET COUNT (X THOUSANDS/UL) AND BLEEDING TIME (SEC) IN CHILD CLASS A, B AND C $(n=64)$

\begin{tabular}{|c|c|c|}
\hline Child Pugh Score & MEAN \pm S.D & ${ }^{*} p$ \\
\hline \multicolumn{3}{|c|}{ Fibrinogen } \\
\hline Class A ( $n=11)$ & $333.90 \pm 45.007$ & A vs $B \quad 0.001$ \\
\hline Class B $(n=26)$ & $261.07 \pm 70.622$ & B vs C 0.002 \\
\hline Class C ( $n=27)$ & $207.33 \pm 38.484$ & A vs C 0.0001 \\
\hline \multicolumn{3}{|c|}{ D-dimer } \\
\hline Class $A(n=11)$ & $195.45 \pm 13.633$ & A vs $B \quad 0.441$ \\
\hline Class B $(n=26)$ & $212.96 \pm 37.845$ & B vs C 0.135 \\
\hline Class C ( $n=27)$ & $234.14 \pm 47.287$ & A vs C 0.022 \\
\hline \multicolumn{3}{|l|}{${ }^{*}$ ANOVA } \\
\hline
\end{tabular}

TABLE III: PLATELET COUNT (X THOUSANDS/UL) AND BLEEDING TIME (SEC) IN CHILD CLASS A, B AND C $(n=64)$

\begin{tabular}{|c|c|c|}
\hline Child Pugh Score & MEAN \pm S.D & ${ }^{*} p$ \\
\hline \multicolumn{3}{|c|}{ Platelet count } \\
\hline Class A $(n=11)$ & $375.36 \pm 39.479$ & A vs $B \quad 0.238$ \\
\hline Class B $(n=26)$ & $343.30 \pm 58.793$ & B vs C 0.0001 \\
\hline Class C $(n=27)$ & $277.03 \pm 55.061$ & A vs C 0.0001 \\
\hline \multicolumn{3}{|c|}{ Bleeding time } \\
\hline Class A $(n=11)$ & $4.63 \pm 1.804$ & A vs B 0.002 \\
\hline Class B $(n=26)$ & $7.88 \pm 2.833$ & B vs C 0.104 \\
\hline Class C ( $n=27)$ & $9.33 \pm 2.496$ & A vs C 0.0001 \\
\hline
\end{tabular}

TABLE IV: PEARSON'S CORRELATION OF CHILD PUGH SCORE WITH DIFFERENT VARIABLES

\begin{tabular}{|l|r|r|}
\hline \multicolumn{1}{|c|}{ Variable } & \multicolumn{1}{c|}{ r- } & \multicolumn{1}{c|}{ p } \\
\hline Prothrombin time & 0.664 & 0.0001 \\
\hline Activated partial thromboplastin time & 0.711 & 0.0001 \\
\hline D-Dimer & 0.348 & 0.005 \\
\hline Bleeding time & 0.533 & 0.0001 \\
\hline Fibrinogen & -0.640 & 0.0001 \\
\hline Platelet count & -0.579 & 0.0001 \\
\hline
\end{tabular}

Fig No. IV-10: Scatter plot showing positive correlation of Prothrombin time with CPC A,B \& C.

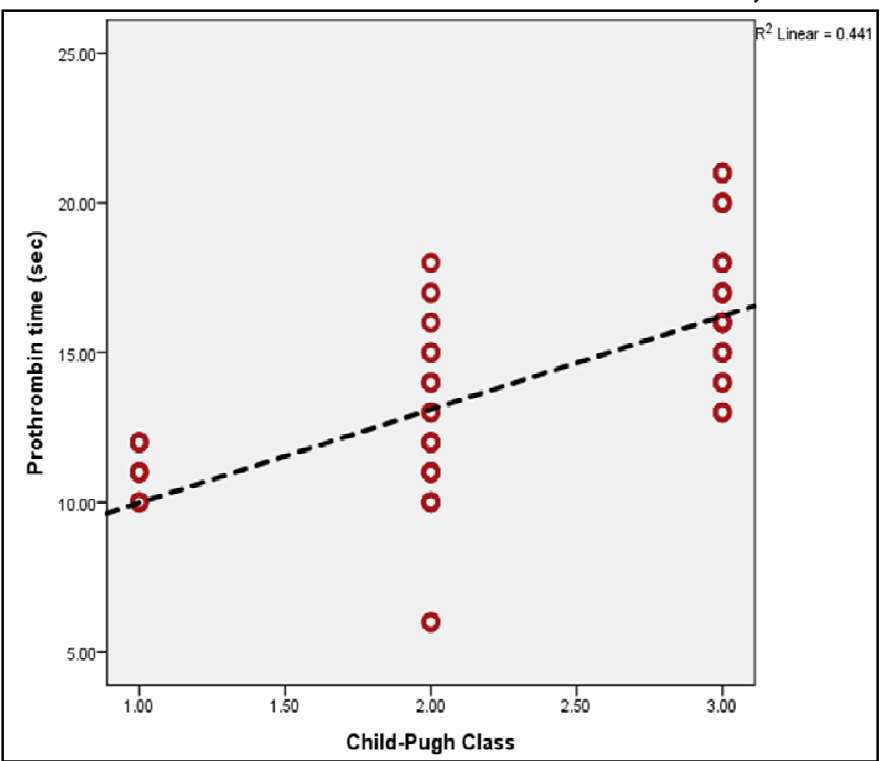

Fig. No. IV-11: Scatter plot showing positive correlation of Activated Partial thromboplastin time with CPC A, B \& C.

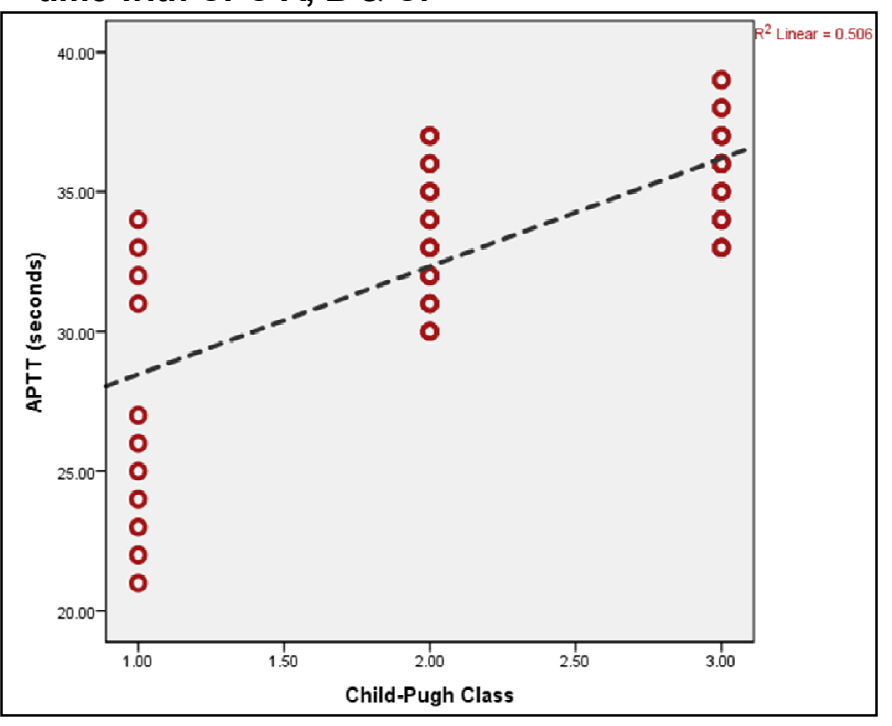


Fig. No. IV-12: Scatter plot showing positive correlation of D-dimer with CPC A, B \& C.

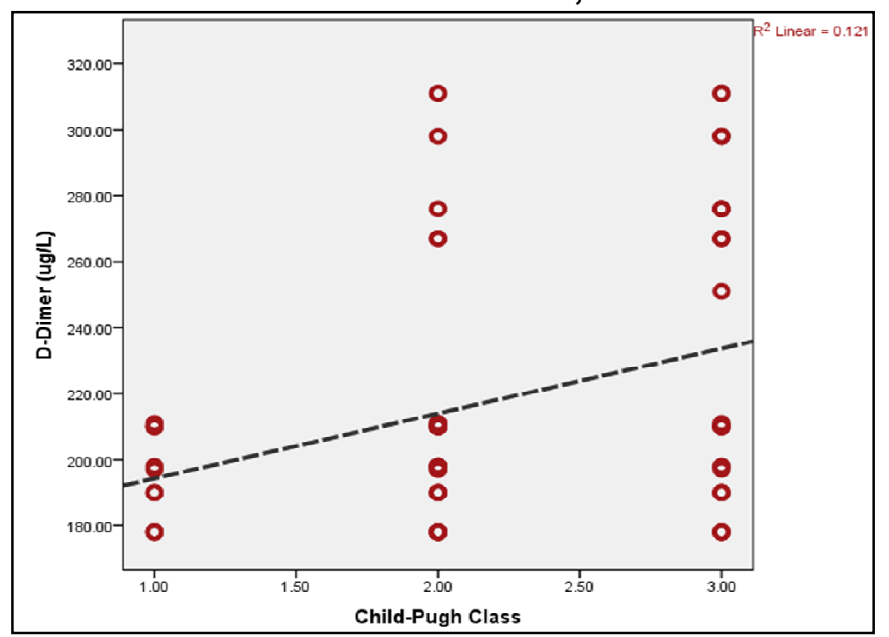

Fig. No. IV-13: Scatter plot showing positive correlation of bleeding time with CPC A, B \& C.

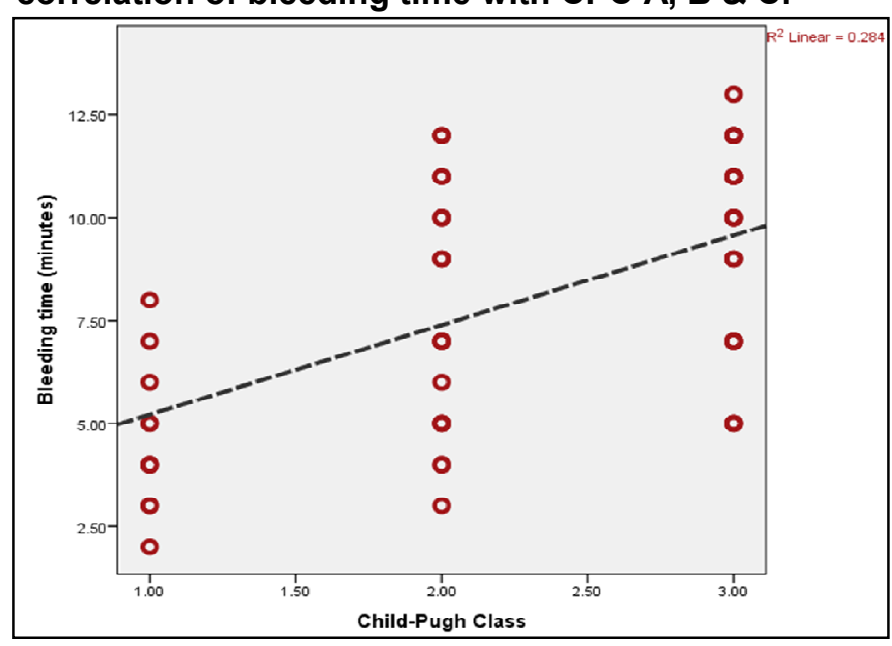

Fig. No. IV-14: Scatter plot showing negative correlation of Fibrinogen with CPC A, B \& C.

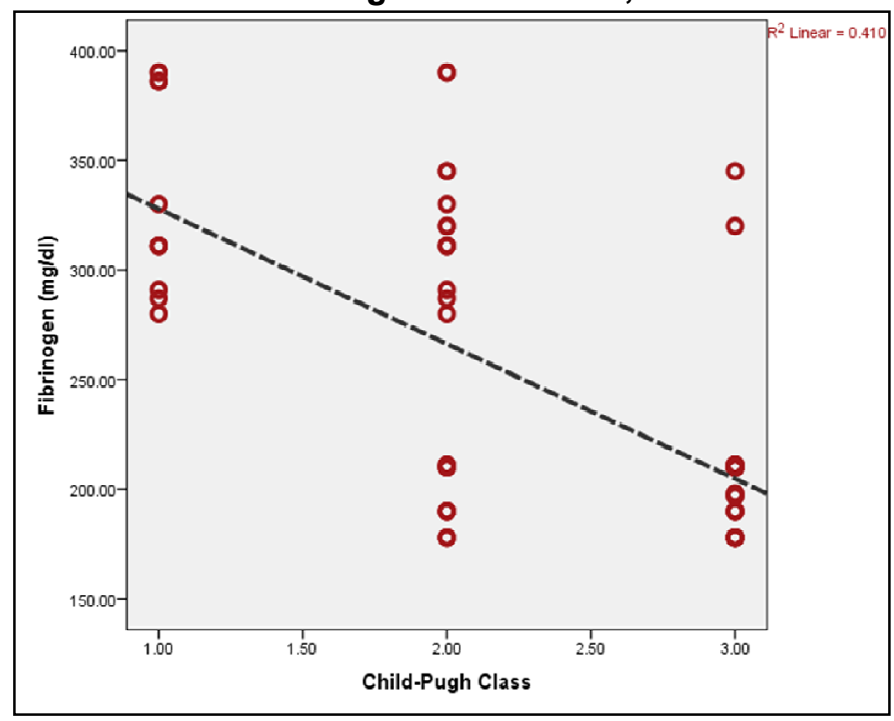

Fig. No. IV-15: Scatter plot showing negative correlation of Platelet count with CPC A, B \& C.

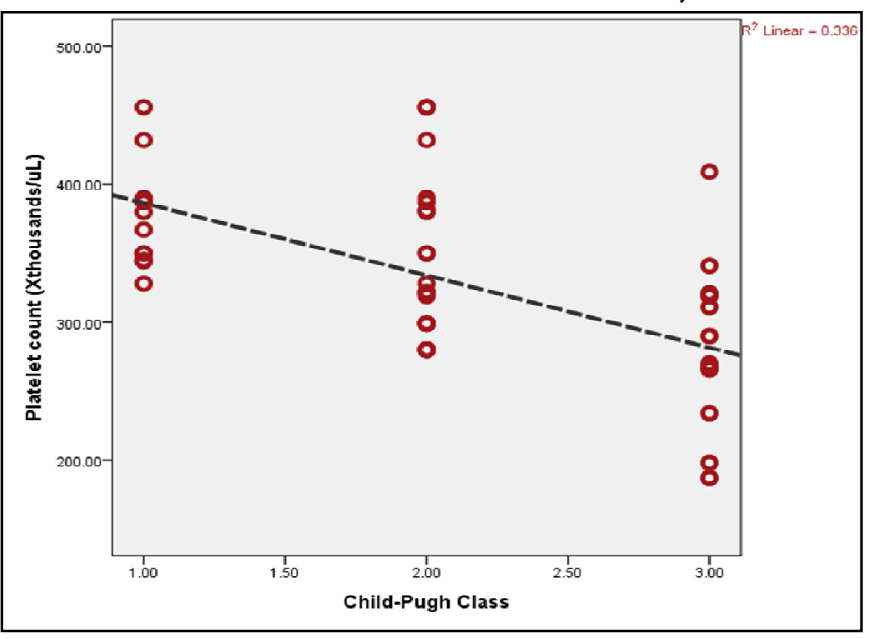

\section{DISCUSSION}

This study was conducted to assess various hemostatic defects in patients with liver cirrhosis and co-relate these with severity of liver damage as assessed by Child Pugh score. Present study included 64 diagnosed patients of Liver Cirrhosis; 47 males and 17 females. These were divided into three groups according to the criteria of Child Pugh score i.e. Group A, B and C. Group A included 11 patients, Group B 26 patients and Group $C$ included 27 patients.

In the present study Mean Platelet count was decreased significantly as compared to normal reference level. The value showed a significant decrease from Child Pugh Score A through C. Similar results were observed by ${ }^{10,11}$, Tripodi $A$, et al $^{10}$ observed that platelet count was decreased in liver cirrhosis but has no co-relation with Child Pugh score . In the present study Mean Bleeding Time was increased significantly as compared to normal reference level. The value showed a significant increase from Child Pugh Score A through C. Similar results were observed by previous study done by Tripodi $A^{12}$. Caldwell SH, et al ${ }^{13}$ reported that Portal hypertension, bacterial infection, endothelial dysfunction and renal failure are central factors denoting to bleeding diathesis with liver disease. In the present study portal hypertension with Ascites was the common finding in majority of the cases. In the present study Mean D-dimer level was increased significantly as compared to normal reference level. The value showed a significant increase from Child Pugh Score A through C. Similar results were observed by Zhang $\mathrm{DL}$, et $\mathrm{al}^{14}$. $\mathrm{D}$-dimer concentration increase with deteriorating liver functions and may be associated with portal vein thrombosis ${ }^{14}$. In the present study Mean Prothrombin Time was increased significantly as compared to 
normal reference level. The value showed a significant increase from Child Pugh Score A through C. Similar results were observed by Agarwal, $\mathrm{S}$, et al and Tripodi $\mathrm{A}$, et $\mathrm{al}^{15,17}$.

Senzolo $M$, et $\mathrm{al}^{11}$ observed that Prothrombin time was decreasing from Child Pugh Score A through C, this was in contrast with the result of the present study.

In the present study Mean Activated partial thromboplastin time was increased significantly as compared to normal reference level. The value showed a significant increase from Child Pugh Score A through $C$. Similar results were observed by Senzolo $\mathrm{M}$, et al and Zhang $\mathrm{DL}$, et $\mathrm{al}^{11,14}$. In the present study Mean Fibrinogen level was decreased significantly as compared to normal reference level. The value showed a significant decrease from Child Pugh Score A through C. Similar results were observed by Senzolo $\mathrm{M}$, et al and Zhang DL, et al ${ }^{11,14}$, therefore finally it is concluded that the haemostatic abnormalities in liver cirrhosis were found to be as significant increase in Prothrombin time, Activated partial thromboplastin time, Bleeding time and D-dimer and Significant decrease in Platelet count and Fibrinogen level. When these hemostatic abnormalities were correlated with Child Pugh Score it was observed that PT, APTT, BT and D-dimer were positively correlated with Child Pugh Score and Fibrinogen level and Platelet count were negatively correlated with Child Pugh Score.

\section{RECOMMENDATIONS}

- As coagulation profile correlates with severity of cirrhosis it should be performed before any surgical procedure.

- To check the hypercoagulability state in cirrhosis further studies are required.

- For assessing the significance of these factors in a greater population sample, for identifying extra danger factors and calculation of the prognostic outcome of anticoagulation therapy and Portosystemic shunting in patients having recognized PVT further studies are needed.

- Causes that disturb primary haemostasis in cirrhotic patients need additional study as they might be essential in managing occasions of bleeding, invasive methods, and surgery, involving liver transplantation.

\section{REFERENCES}

1. Lawrence SF. Liver, Biliary Tract and Pancreatic Disorders. In Maxine AP, Stephen JM, eds. Current Medical Diagnosis and Treatment. $53^{\text {rd }}$ ed. McGrew Hill Education USA; 2014: Pp-665.

2. Anstee $\mathrm{QM}$, Jones DEJ. Liver and biliary tract disease. In: Walker BR, Colledge NR, Ralston SH, Perman ID, eds. Davidson's Principles and Practice of Medicine. 22nd ed. Philadelphia, PA: Elsevier Churchill Livingstone; 2012:chap 23.

3. Kumar P, Clark M. Liver, Biliary Tract and Pancreatic Disease. In Kumar P, Clark M , eds. Clinical Medicine Eighth Edition, Saunders Elsevier; 2012: Pp 329 .

4. Mucino-Bermejo J, Carrillo-Esper R, Uribe M, Mendes-Sanchez N. Coagulation abnormalities in the Cirrhotic Patient. Annals Of Hepatology , 2013; 12 (5): 713-724.

5. Saja MF, Abdo AA, Sanai FM, Shaikh SA, Gader AG. The Coagulopathy of Liver Disease: Does Vitamin K Help? Blood Coagul Fibrinolysis 2012; 24(1):10-17.

6. Schaden E, Saner Fh, Gorlinger K. Coagulation Pattern In Crit-Ical Liver Dysfunction. Curr Opin Crit Care 2013; 19: 142-8.

7. Tripodi A, Primignani $M$, Chantarangkul V, et al. Global Hemostasis tests in patients with Cirrhosis before and after prophylactic platelet transfusion. Liver Int 2013; 33(3): 362-7.

8. Shah NL, Northup P, Caldwell S. A clinical survey of bleeding, thrombosis, and blood product use in decompensated cirrhosis patients. Annals of Hepatology. 2012; 11 (5): 686-90

9. Ying Peng, Xingshun Qi, Junna Dai, Hongyu Li, Xiaozhong Guo. Child-Pugh versus MELD score for predicting the in-hospital mortality of acute upper gastrointestinal bleeding in liver cirrhosis. Int J Clin Exp Med. 2015; 8(1): 751-7.

10. Tripodi A, Primignani M, Mannucci PM. Abnormalities of hemostasis and bleeding in chronic liver disease: The Paradigm is Challenged. Intern Emerg Med 2010; 5(1):7-12.

11. Senzolo M, Rodriguez-Castro KI, Rossetto V, et al. Simioni. Increased anticoagulant response to low-molecular-weight heparin in plasma from patients with advanced cirrhosis. J Thromb Haemost. 2012.10(9):1823-9.

12. Tripodi A, Salerno F, Chantarangkul V, et al. Evidence of normal thrombin generation in cirrhosis despite abnormal conventional coagulation tests. Hepatology 2005;41(3):553-8.

13. Caldwell SH, Hoffman, M, Lisman $\mathrm{T}$, et al. Coagulation disorders and hemostasis in liver disease: pathophysiology and critical assessment of current management . Hepatology.2006. 44 (4):1039-46.

14. Zhang DL, Hao JY, Yang N. Value of D-dimer and protein $S$ for diagnosis of portal vein thrombosis in patients with liver cirrhosis. J Int Med Res 2013; 41(3):664-72.

15. Agarwal S, Joyner KA Jr, Swaim MW. Ascites 
fluid as a possible origin for hyperfibrinolysis in advanced liver disease. Am J Gastroenterol 2000; 95(11):3218-24.

16. Toschi V, Rocchini GM, Motta A, et al. The hyperfibrinolytic state of liver cirrhosis: possible pathogenetic role of ascites. Biomed Pharmacother. 1993;47:345-52.

17. Tripodi A, Primignani M, Chantarangkul V, et al. Thrombin generation in patients with cirrhosis: the role of platelets. Hepatology 2006;44(2):440-5.

\begin{tabular}{|l|}
\hline AUTHOR AFFILIATION: \\
Dr. Hanozia Hassan \\
Assistant Professor, Department of Physiology \\
Bilawal Medical College Jamshoro, Sindh-Pakistan. \\
Dr. Asmat Kamal Ansari (Corresponding Author) \\
Professor, Department of Physiology \\
Bilawal Medical College Jamshoro, Sindh-Pakistan. \\
Email: asmat.ansari5@gmail.com \\
Dr. Salma Farrukh Memon \\
Assistant Professor, Department of Physiology \\
Liaquat University of Medical \& Health Sciences \\
Jamshoro, Sindh-Pakistan.
\end{tabular}

